\title{
7ND protein exerts inhibitory effects on both osteoclast differentiation in vitro and lipopolysaccharide-induced bone erosion in vivo
}

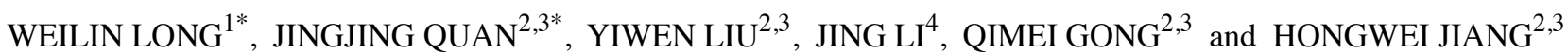 \\ ${ }^{1}$ Department of Stomatology, The Third Affiliated Hospital of Sun Yat-sen University, Guangzhou, Guangdong 510630; \\ ${ }^{2}$ Department of Operative Dentistry and Endodontics, Guanghua School of Stomatology, Hospital of Stomatology, \\ Sun Yat-sen University, Guangzhou, Guangdong 510055; ${ }^{3}$ Guangdong Provincial Key Laboratory of Stomatology, \\ Guanghua School of Stomatology, Hospital of Stomatology, Sun Yat-sen University, Guangzhou, \\ Guangdong 510080; ${ }^{4}$ Department of Stomatology, Affiliated Longhua People's Hospital, \\ Southern Medical University, Shenzhen, Guangdong 518000, P.R. China
}

Received August 28, 2018; Accepted February 25, 2020

DOI: $10.3892 / \mathrm{mmr} .2020 .11119$

\begin{abstract}
Excessive numbers of osteoclasts are responsible for inflammation-induced osteolysis. Identification of osteoclast-targeting agents may facilitate the development of a novel therapeutic approach for the treatment of pathological bone loss. Seven-amino acid truncated (7ND) protein, a mutant form of monocyte chemoattractant protein-1 (MCP-1), functions as a competitive inhibitor of MCP-1. However, the effects of 7ND protein on osteoclast differentiation remain unknown. Therefore, in the present study, the effects of 7ND protein on osteoclast differentiation induced by tumour necrosis factor superfamily member 11 were investigated. In the present study, 7ND protein inhibited the osteoclast differentiation of peripheral blood mononuclear cells without influencing cell proliferation. Furthermore, to evaluate the effects of 7ND protein in vivo, a lipopolysaccharide (LPS)-induced calvarial bone erosion animal model was established. The 7ND protein remarkably attenuated LPS-induced bone resorption, as assessed by micro-computed tomography and histological analysis. Taken together, the present results suggested the feasibility of local delivery of $7 \mathrm{ND}$ protein to mitigate osteoclast differentiation
\end{abstract}

Correspondence to: Professor Qimei Gong or Professor Hongwei Jiang, Department of Operative Dentistry and Endodontics, Guanghua School of Stomatology, Hospital of Stomatology, Sun Yat-sen University, 56 West Lingyuan Road, Guangzhou, Guangdong 510055, P.R. China

E-mail: gongqim@mail.sysu.edu.cn

E-mail: jianghw@163.com

*Contributed equally

Key words: monocyte chemoattractant protein-1, seven-amino acid truncated protein, osteoclast, lipopolysaccharide, osteolysis and LPS-induced osteolysis, which may represent a potential approach to treat inflammatory bone destruction.

\section{Introduction}

Bone development and maintenance are regulated by highly dynamic remodelling processes that are influenced by bone-degrading osteoclasts, bone-forming osteoblasts and mechanical-sensing osteocytes (1). Osteoclasts are the only cell type able to remove bone tissue, and they are responsible for both physiological and pathological bone resorption, which is indispensable during skeletal development and maintenance (2). Osteoclasts are multinucleated cells that are formed by the fusion of myeloid hematopoietic precursors formed in the bone marrow (3). Osteoclast precursors are circulating blood monocytes that are recruited to sites of bone remodelling where they fuse into osteoclasts following specific stimuli (4). Therefore, identifying agents capable of suppressing osteoclast differentiation may facilitate the development of a therapeutic strategy for the treatment of pathological bone loss.

Bacteria and their by-products can induce the release of pro-inflammatory cytokines, such as macrophage chemoattractant protein-1 (MCP-1), tumour necrosis factor- $\alpha$ (TNF- $\alpha$ ), interleukins (ILs) and prostaglandin E2, which directly or indirectly stimulate osteoclast differentiation $(5,6)$. In a previous study, Jiang et al (7) showed the relationship between inflammatory cytokines and bone resorption. Macrophage-derived chemokines induce macrophages and osteoclast precursor cells to polarize and migrate to inflammatory tissues, whereas inflammatory cytokines promote osteoclast differentiation and suppress osteoblast formation.

Among various inflammatory cytokines, MCP-1 is one of the most abundantly released and is responsible for macrophage cell recruitment and activation during acute inflammation, promoting multinuclear cells to fuse into osteoclasts $(8,9)$. Seven-amino acid truncated (7ND) protein is a mutant version of the MCP-1 protein. The 7ND protein lacks seven amino 
acids between position 2 and 8 of MCP-1, located in the $\mathrm{N}$-terminus and functions as a dominant negative inhibitor of MCP-1 $(6,10)$. In a previous study, Yao et al (6) showed that n7ND protein effectively decreased $\mathrm{MCP}-1$-induced migration of THP-1 macrophages in vitro. In addition, n7ND protein injection or delivery from a layer-by-layer coating platform significantly inhibited wear particle-induced osteolysis in vivo $(7,11)$. However, the efficacy of $7 \mathrm{ND}$ on human TNF superfamily member 11 (TNFSF11)-induced osteoclast differentiation and lipopolysaccharide (LPS)-induced osteolysis has yet to be demonstrated. Therefore, in the present study, the effects of 7ND proteins on human peripheral blood mononuclear cell (PBMC) proliferation and osteoclast differentiation were investigated in vitro, and the ability of 7ND to decrease LPS-induced bone erosion was examined in vivo.

\section{Materials and methods}

Reagents. Recombinant human colony-stimulating factor 1 (rhCSF1), TNFSF11 (rhTNFSF11) and MCP-1 (rhMCP-1) were purchased from PeproTech, Inc. $\alpha$-minimal essential medium ( $\alpha$-MEM), penicillin-streptomycin solution, PBS without calcium and magnesium, FBS, DAPI staining solution and Rhodamine-conjugated phalloidin were purchased from Gibco; Thermo Fisher Scientific, Inc. The tartrate-resistant acid phosphatase (TRAP) staining kit, Escherichia coli (E. coli) LPS, and haematoxylin and eosin (H\&E) were purchased from Sigma-Aldrich; Merck KGaA. Cell Counting Kit-8 (CCK-8) reagent was obtained from Dojindo Molecular Technologies, Inc. 7ND protein was purified as previously reported (12).

PBMC isolation. Heparinized blood $(100 \mathrm{ml})$ was collected from each healthy volunteer (4 males and 5 females; 25-35 years of age; recruitment date from September 2016 to January 2018) at the Department of Operative Dentistry and Endodontics at the Affiliate Stomatology Hospital of Sun Yat-sen University. To be included volunteers had to be Chinese, healthy, 30 years of age and medication-free for one month. The patients provided written informed consent. The study was approved by The Research Ethics Committee of Guanghua School of Stomatology, Affiliated Stomatological Hospital, Sun Yat-sen University. Blood was collected in BD vacutainer cell preparation tubes (CPT; BD Biosciences) with $0.100 \mathrm{M}$ sodium citrate. Heparinized blood was centrifuged at $1,500 \mathrm{x} \mathrm{g}$ and $0^{\circ} \mathrm{C}$ for $30 \mathrm{~min}$, and the cell layer above the Ficoll-Paque formed in the CPT was collected, resuspended in $10 \mathrm{ml}$ of $\alpha$-MEM and recentrifuged at $195.65 \mathrm{x} \mathrm{g}$ and $37^{\circ} \mathrm{C}$ for $5 \mathrm{~min}$. Then, the cells were cultured in $0.5 \mathrm{ml}$ of $\alpha$-MEM supplemented with $10 \%$ FBS and $1 \%$ penicillin-streptomycin solution in a 24 -well plate at a density of $3 \times 10^{5}$ cells/well and incubated at $37^{\circ} \mathrm{C}$ in a humidified atmosphere with $5 \% \mathrm{CO}_{2}$ for 1 day. On the second day, the complete medium was replaced, floating cells were discarded, and the adherent cells were identified as PBMCs (12). PBMCs were cultured and used for subsequent experiments.

Cell viability assay. PBMCs were incubated at $37^{\circ} \mathrm{C}$ in 96-well plates at a density of $1 \times 10^{5}$ cells/well for 1 day, the complete medium was replaced and increasing concentrations of $7 \mathrm{ND}$ protein $(0-100 \mathrm{ng} / \mathrm{ml})$ were added. After 1,2 and
3 days, CCK-8 reagent (10 $\mu \mathrm{l})$ was added to each well followed by incubation at $37^{\circ} \mathrm{C}$ in the dark for $3 \mathrm{~h}$, according to the manufacturer's protocols. The absorbance was measured at $450 \mathrm{~nm}$ using a microplate reader (BioTEK Instruments, Inc.).

Osteoclast differentiation and identification. PBMCs were plated in 24-well plates and supplemented with rhCSF1 $(25 \mathrm{ng} / \mathrm{ml})$ and $\mathrm{rhTNFSF} 11(40 \mathrm{ng} / \mathrm{ml})$ to induce osteoclast differentiation. Groups were arranged as follows: i) Untreated monocytes; ii) monocytes treated with rhCSF1 (25 ng/ml) and rhTNFSF11 (40 ng/ml); iii) monocytes treated with rhCSF1 (25 ng/ml), rhTNFSF11 (40 ng/ml) and rhMCP-1 (25 ng/ml); and iv) monocytes treated with rhCSF1 (25 ng/ml), rhTNFSF11 (40 $\mathrm{ng} / \mathrm{ml})$ and 7ND protein $(25 \mathrm{ng} / \mathrm{ml})$. The medium and all cytokines were replaced every 3 days for 15 days, and osteoclasts were cultured as previously described $(13,14)$.

For TRAP staining, cells were fixed in $4 \%$ paraformaldehyde for $20 \mathrm{~min}$ at room temperature $\left(18-26^{\circ} \mathrm{C}\right)$ and then stained with TRAP staining solution at $37^{\circ} \mathrm{C}$ for $1 \mathrm{~h}$, according to the manufacturer's protocol (Sigma-Aldrich; Merck KGaA). The images were captured by a light microscope (Carl Zeiss AG) using magnification, x100. TRAP-positive cells with $\geq 3$ nuclei were considered multinucleated osteoclasts.

For actin cytoskeleton staining, cells were washed with PBS three times, permeabilized with $0.2 \%$ Triton $\mathrm{X}-100$ in PBS for $10 \mathrm{~min}$ and stained with fluorescent phalloidin (1:200) diluted in PBS for $20 \mathrm{~min}$ at room temperature $\left(18-26^{\circ} \mathrm{C}\right)$. Nuclei counterstaining was performed by DAPI (1:500) for $5 \mathrm{~min}$ at room temperature $\left(18-26^{\circ} \mathrm{C}\right)$, followed by fluorescence microscopy (Carl Zeiss AG) using magnification, x100. In total, four fields were randomly selected, and the numbers of osteoclasts and filamentous (F)-actin rings were counted by two independent investigators.

In vivo animal model of bone loss by LPS. A total of 78 five-week-old male C57BL/6J mice (16.3-18.3 g) were purchased from The Animal Resources Center of Sun Yat-sen University and housed at a controlled temperature $\left(22-24^{\circ} \mathrm{C}\right)$ and humidity (55-60\%) under a 12-h light/dark cycle with free access to food and water. All protocols were reviewed and approved by the Sun Yat-sen University Ethics Committee (approval nos. IACUC-DB-17-1105 and IACUC-DD-17-1112).

To establish an LPS-induced osteolysis murine model, the mice were randomly divided into three groups ( $n=20 /$ group) and injected with E. coli LPS or PBS subcutaneously, over the calvaria. Groups were arranged as follows: i) Control group injected with PBS; ii) mice injected with $5 \mathrm{mg} / \mathrm{kg}$ LPS; and iii) mice injected with $25 \mathrm{mg} / \mathrm{kg}$ LPS. Animals were monitored every day and the weight of each mouse was recorded on the last experimental day. In total, five mice from each group were sacrificed on day 1, 3,5 and 7, and all skull bones were dissected and soaked in $75 \%$ ethanol at room temperature $\left(18-26^{\circ} \mathrm{C}\right)$ for micro-computed tomography $(\mu$-CT) analysis.

To study the function of $7 \mathrm{ND}$ protein in vivo, the mice were injected subcutaneously over the calvaria and randomly divided into three groups ( $n=6$ in each group): i) Negative control group injected with PBS on day 1 and day 3; ii) Positive control group injected with LPS $(25 \mathrm{mg} / \mathrm{kg})$ on day 1 and PBS on day 3; and iii) Experimental group injected with LPS $(25 \mathrm{mg} / \mathrm{kg})$ and $7 \mathrm{ND}$ protein $(3 \mu \mathrm{g}$ in $100 \mu \mathrm{l} \mathrm{PBS})$ on day 1 and 
A
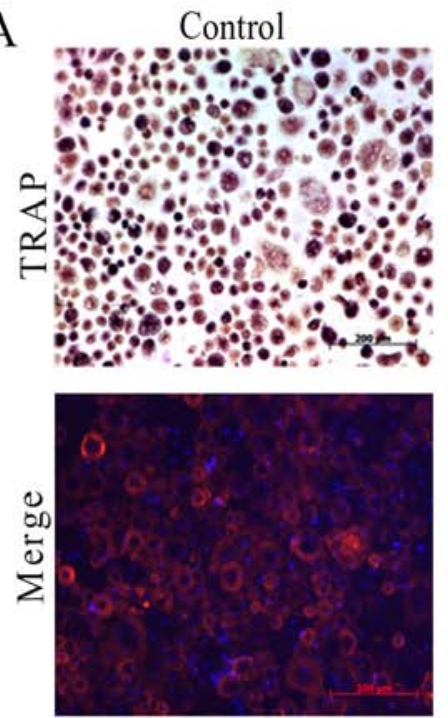

$\mathrm{B}$

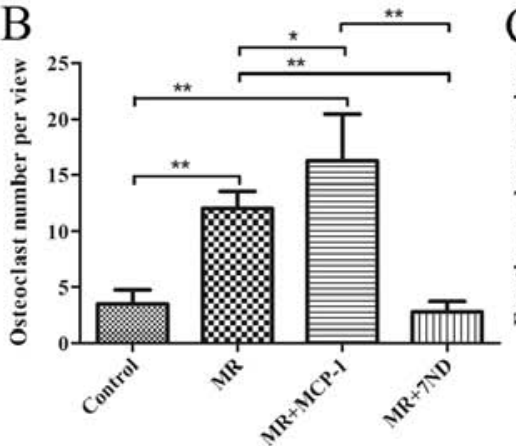

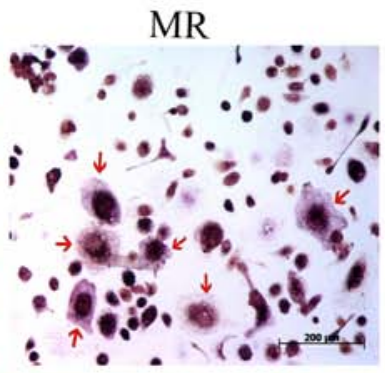
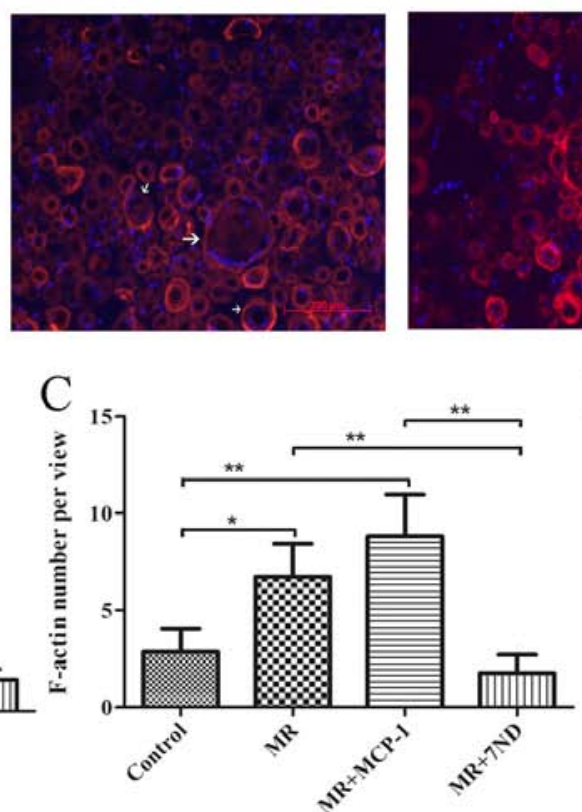

MR+MCP-1
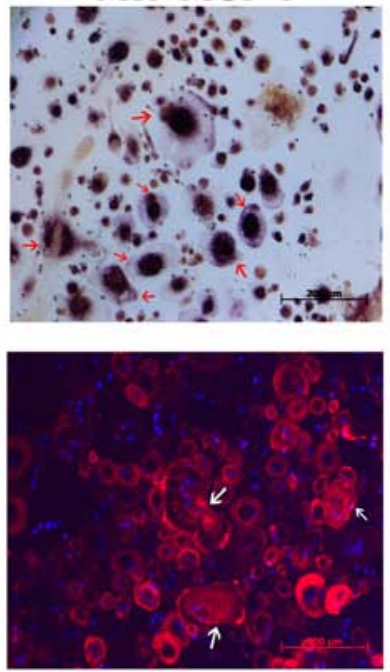

$\mathrm{D}$

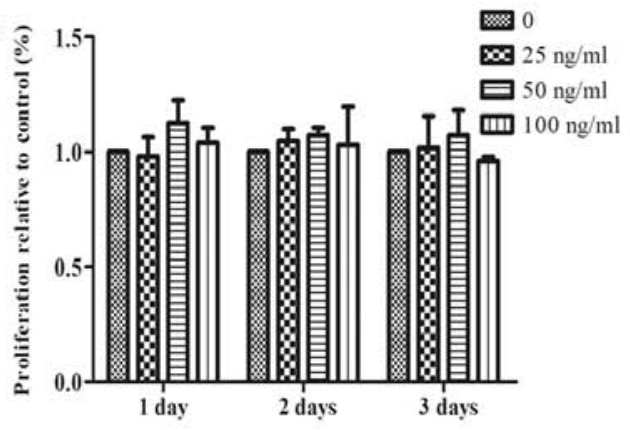

Figure 1. 7ND protein inhibits osteoclast differentiation without influencing PBMC proliferation. (A) 7ND protein treatment inhibited osteoclast differentiation, assessed by TRAP and immunofluorescence staining. Nuclei are stained by DAPI in blue and F-actin rings by phalloidin in red. Red arrows indicate TRAP-positive cells. Blue arrows indicate F-actin rings. Scale bar, $200 \mu \mathrm{m}$. Quantification of (B) osteoclast numbers and (C) F-actin ring numbers. (D) Cell Counting Kit-8 assay showed that the 7ND protein exhibited no significant effects on PBMC proliferation. Proliferation results were normalized to the viability of untreated cells. ${ }^{*} \mathrm{P}<0.05,{ }^{* *} \mathrm{P}<0.01$. MR, cells treated with colony-stimulating factor $1+$ tumour necrosis factor superfamily member $11 ; 7 \mathrm{ND}$, seven-amino acid truncated; TRAP, tartrate-resistant acid phosphatase; MCP-1, macrophage chemoattractant protein-1; F-actin, filamentous actin; PBMC, peripheral blood mononuclear cell.

7ND protein ( $3 \mu \mathrm{g}$ in $100 \mu \mathrm{l}$ PBS) without LPS on day 3. The animals were sacrificed on day 6 , and their skull bones were dissected and soaked in $75 \%$ ethanol at room temperature $\left(18-26^{\circ} \mathrm{C}\right)$ for $\mu$-CT analysis.

$\mu$-CT imaging and analysis. All calvaria were scanned by a $\mu$-CT scanner ( $\mu$ CT50; SCANCO Medical AG). The scanning parameters were $8 \mathrm{~W}, 70 \mathrm{kV}, 114 \mu \mathrm{A}, 360^{\circ}$ rotation and a pixel size of $20 \mu \mathrm{m}$. After scanning, VGStudio Max software (version 1.2.1; Volume Graphics $\mathrm{GmbH}$ ) was used to reconstruct the three-dimensional (3D) images. Subsequently, the $3 \mathrm{D}$ images were converted into graphic images by a blinded observer. To study the murine LPS-induced osteolysis model, the resorption areas of a region of interest in the frontal and parietal bones were defined with Adobe Photoshop (version 12.0.3; Adobe Systems, Inc.) by two independent investigators. ImageJ software (version 6.0; National Institutes of Health) was used to analyse the images of resorption areas and to determine the percentage of the calvarial resorption area of each sample.

For quantitative analysis of the function of 7ND protein with LPS-induced osteolysis, the software of $\mu$ CT50 (SCANCO Medical AG) was used to analyse $4 \mathrm{~mm}$ thick (200 slices) skull sections from the midline suture of the skull in its centre to calculate bone mineral density (BMD), bone volume (BV) and the bone volume per tissue volume (BV/TV). The skulls were then fixed in $4 \%$ paraformaldehyde at room temperature $\left(18-26^{\circ} \mathrm{C}\right)$ for 1 day for histological analysis.

Histological evaluation of osteolysis. All skulls were fixed in $4 \%$ paraformaldehyde for 1 day at room temperature $\left(18-26^{\circ} \mathrm{C}\right)$ and the soft tissue was removed. The remaining calvaria bones were decalcified for 3 weeks at room temperature $\left(18-26^{\circ} \mathrm{C}\right)$

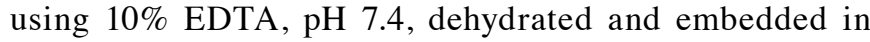
paraffin. Serial sections of $5-\mu \mathrm{m}$ thickness were cut by a Leica microtome RM2255 (Leica Microsystems GmbH), including the centre of the calvaria, the site of drug injection. For histological examination, the slides were stained with $\mathrm{H} \& \mathrm{E}$ at room temperature $\left(18-26^{\circ} \mathrm{C}\right)$ and TRAP at $37^{\circ} \mathrm{C}$ for $1 \mathrm{~h}$ according to the manufacturer's protocols. Images in six randomly selected fields were captured by a light microscope (Carl Zeiss AG) at magnification, x100. TRAP-positive osteoclasts in the skull bones were counted by two independent investigators.

Statistical analysis. Each experiment was performed $>3$ times. All statistical analyses were performed using SPSS (version 20; 

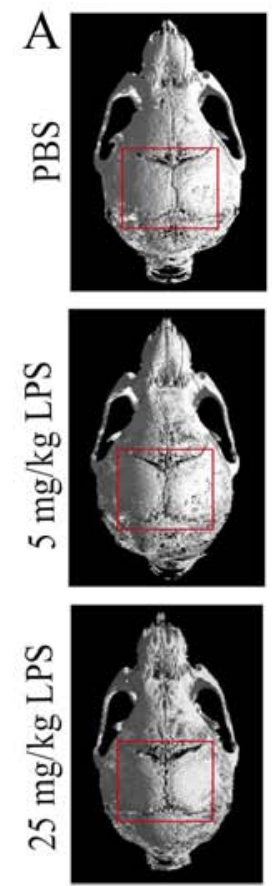

1 day
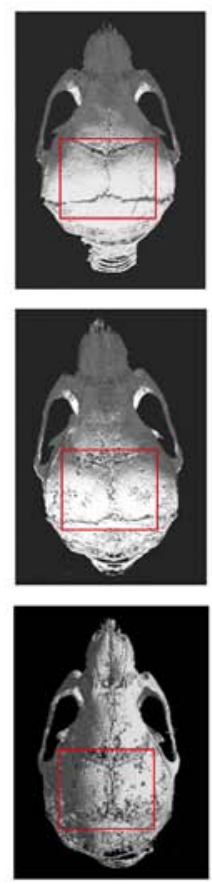

3 days
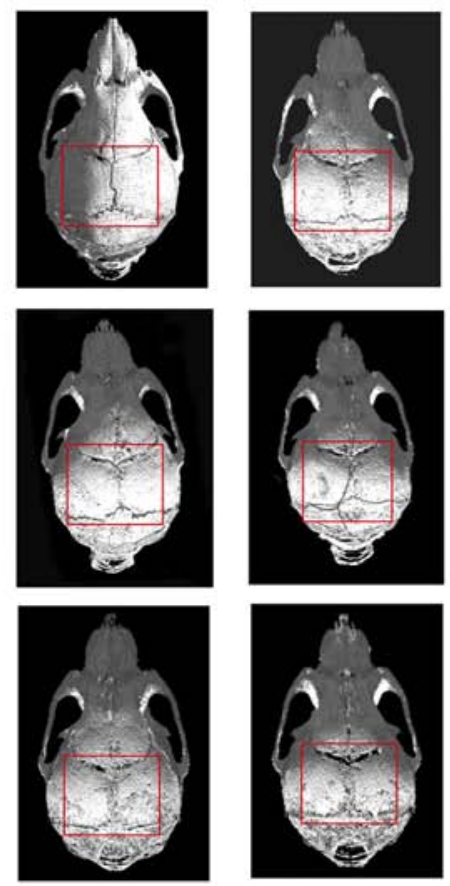

5 days

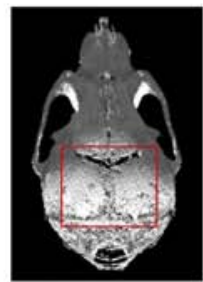

7 days

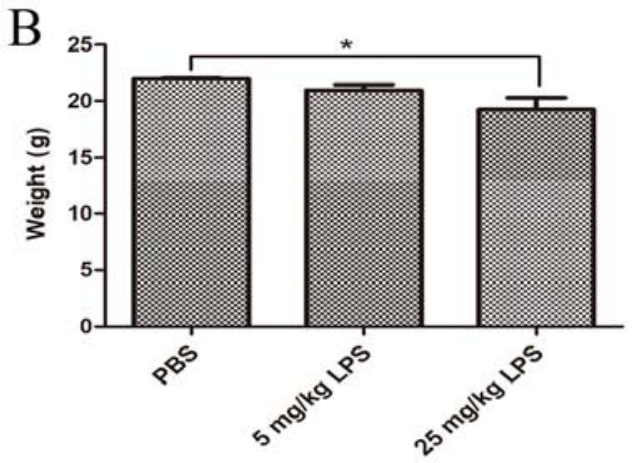

C

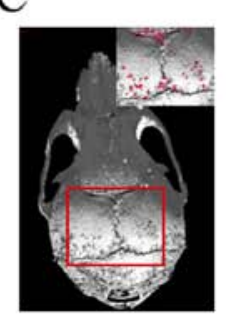

$\mathrm{D}$

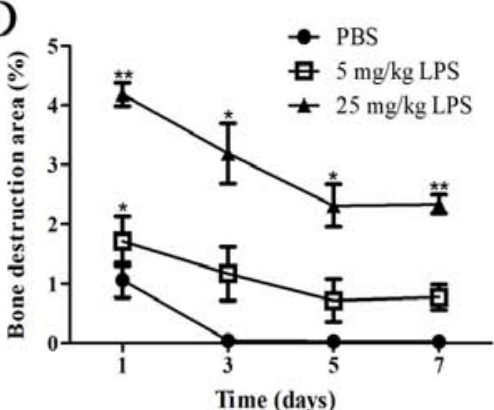

E

PBS

$5 \mathrm{mg} / \mathrm{kg}$ LPS
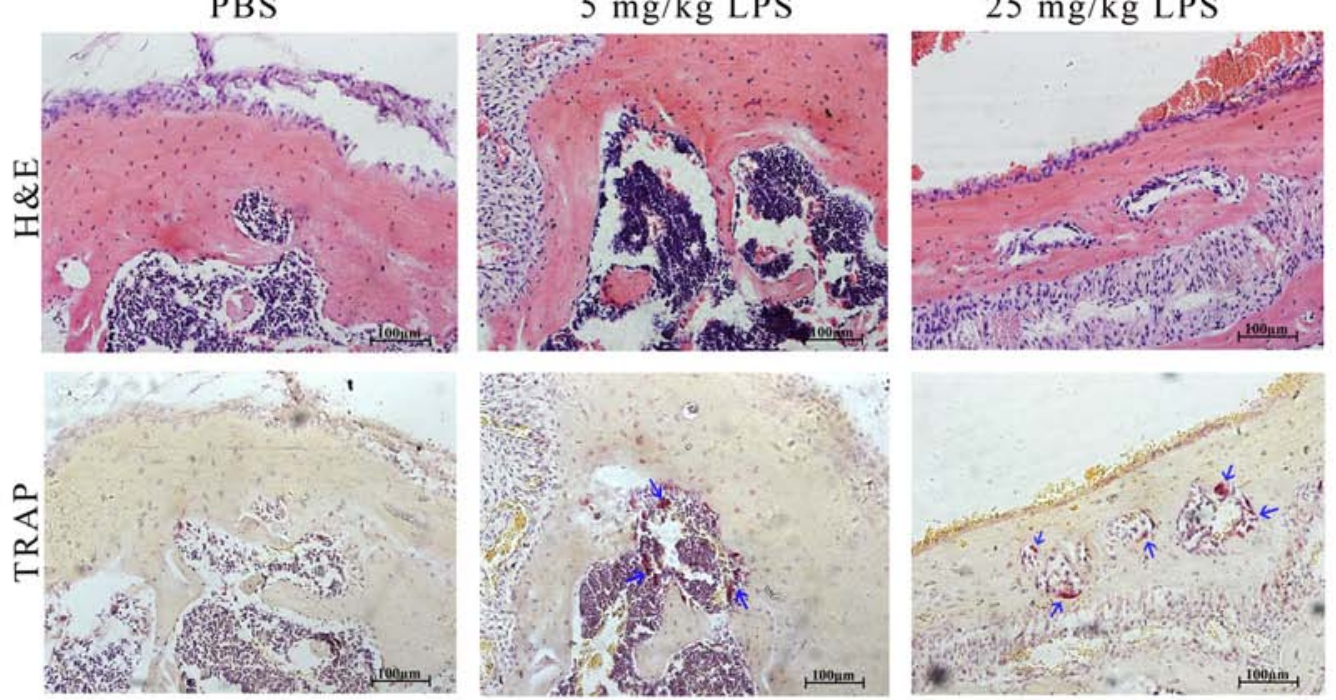

Figure 2. Establishment of an in vivo mouse model of bone loss by LPS injection. (A) Images of calvaria collected from mice on days $1,3,5$ and 7 after local subcutaneous injection of PBS, and 5 or $25 \mathrm{mg} / \mathrm{kg}$ LPS. (B) Mouse weight. $\mathrm{n}=5$. ${ }^{*} \mathrm{P}<0.05$. (C) Region of interests are indicated by red boxes, showing the area of bone destruction. (D) Percentage of the bone destruction area. ${ }^{*} \mathrm{P}<0.05,{ }^{* *} \mathrm{P}<0.01$ vs. PBS. (E) H\&E and TRAP staining were used to stain osteoclasts. Blue arrows indicate osteoclast-like cells. scale bar, $100 \mu \mathrm{m}$. LPS, lipopolysaccharide; H\&E, haematoxylin and eosin; TRAP, tartrate-resistant acid phosphatase.

IBM Corp.). Data are presented as the mean \pm SD. Data were evaluated for a normal distribution using Shapiro-Wilk test. The data were normally distributed, and the groups were compared using one-way ANOVA followed by Bonferroni's test. $\mathrm{P}<0.05$ was considered to indicate a statistically significant difference.

\section{Results}

$7 N D$ protein inhibits osteoclast differentiation in PBMCs. PBMCs were induced to differentiate into osteoclasts in the positive control group by adding rhCSF1 and rhTNFSF11 to the culture for 15 days (Fig. 1A). The $7 \mathrm{ND}$ protein $(25 \mathrm{ng} / \mathrm{ml})$ was added in osteoclast differentiation media, and TRAP staining revealed that significantly fewer osteoclasts formed in the presence of $7 \mathrm{ND}$ protein. In contrast, significantly more osteoclasts formed in the presence of rhMCP-1 (Fig. 1A and B). Immunofluorescence results suggested similar results, with significantly decreased F-actin signal in osteoclasts in the presence of the 7ND protein (Fig. 1A and C). Compared with the differentiation media, a decreased number of osteoclasts were observed in the negative control group (Fig. 1A-C).

$7 N D$ protein does not affect PBMC proliferation. A CCK-8 assay was performed to determine the proliferation of 

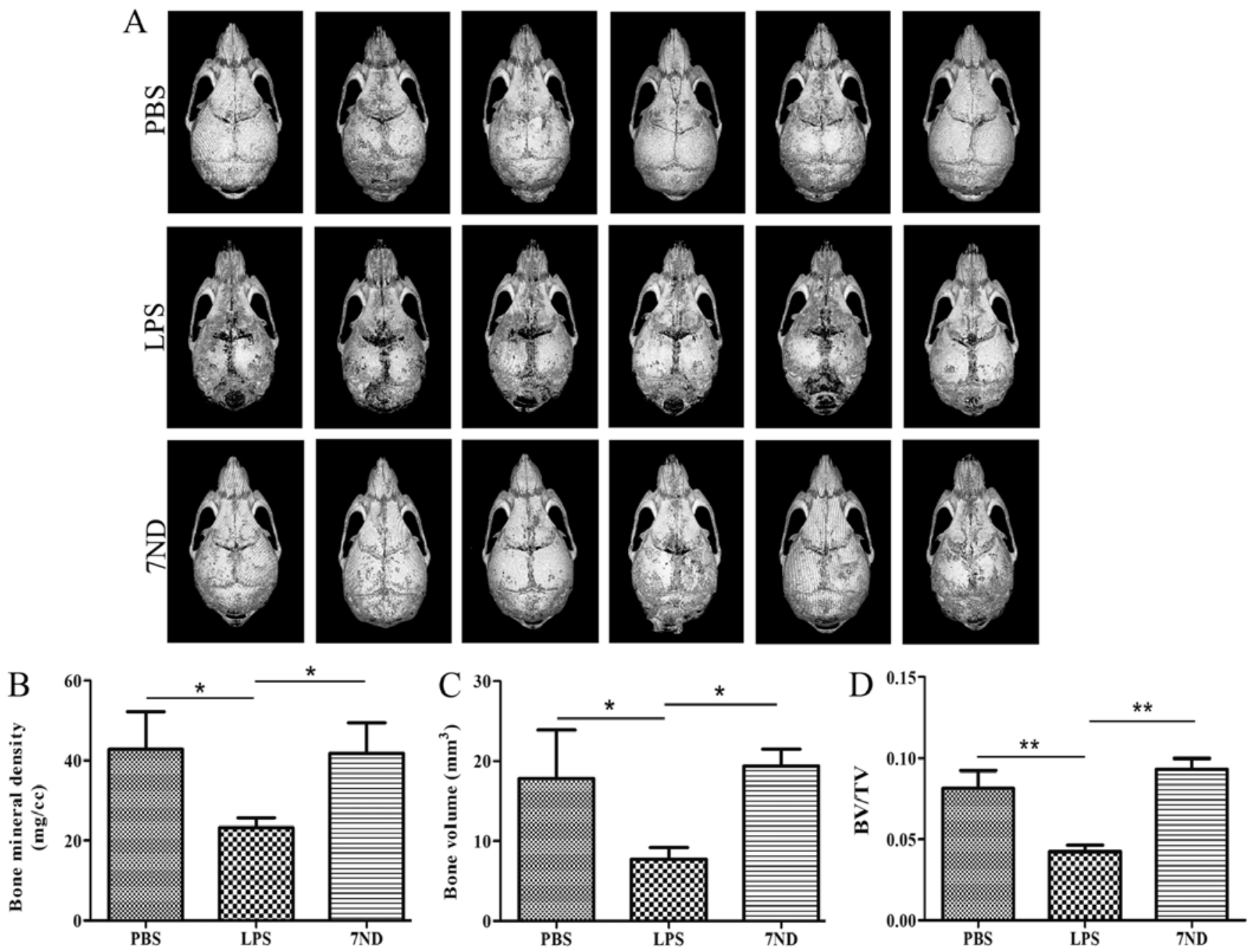

Figure 3. 7ND treatment decreases LPS-induced osteolysis in the calvaria. (A) Micro-computed tomography images of an LPS-induced bone resorption murine calvaria model following subcutaneous injection with PBS and 7ND. 7ND protein decreased LPS-induced osteolysis in C57BL/6J mice. Quantification of (B) BMD, (C) BV and (D) BV/TV. LPS group exhibited statistically lower BMD, BV and BV/TV. 7ND treatment exhibited no significant differences compared with the PBS group. N=6/group. "P<0.05; ${ }^{* *} \mathrm{P}<0.01$. 7ND, seven-amino acid truncated; BV, bone volume; BMD, bone mineral density; BV/TV, bone volume per tissue volume; LPS, lipopolysaccharide.

PBMCs treated with 7ND protein. The results indicated that treatment with $7 \mathrm{ND}$ protein at various concentrations (0-100 $\mathrm{ng} / \mathrm{ml})$ for 1-3 days did not affect PBMC proliferation (Fig. 1D).

Establishment of a mouse LPS-induced bone loss model. $\mu$-CT imaging confirmed that LPS injection induced osteolysis. Increasing concentrations of LPS were associated with more defects in the entire calvarial bone (Fig. 2A). The defects were indicated by the percentage of bone destruction area, and osteolysis induced by $25 \mathrm{mg} / \mathrm{kg}$ LPS was found to last longer compared with $5 \mathrm{mg} / \mathrm{kg}$ LPS (Fig. 2A,C and D). The weights of the mice indicated the health status of the mice (Fig. 2B). H\&E and TRAP staining of calvarial bones confirmed that LPS was able to induce osteoclast differentiation (Fig. 2E). Collectively, the percentage of the bone destruction area and the weights of the mice showed that a high concentration $(25 \mathrm{mg} / \mathrm{kg}$ ) of LPS induced osteolysis that was detected for $>5$ days, whereas a low concentration $(5 \mathrm{mg} / \mathrm{kg})$ of LPS induced significant osteolysis that was detected for 1 day.
$\mu-C T$ imaging and analysis indicates that $7 N D$ protein decreases osteolysis. $\mu$-CT imaging suggested that 7ND protein injection reduced LPS-induced bone resorption. Bone defects decreased in the entire calvarial bone following treatment with 7ND protein compared with the LPS group (Fig. 3A). BMD, BV and BV/TV were used to measure the degree of bone loss. After treatment with 7ND, BMD, BV and BV/TV were significantly increased compared in the LPS group (Fig. 3B-D). Importantly, treatment with 7ND did not present significant differences compared with the PBS group (Fig. 3B-D), suggesting that 7ND protein significantly inhibited osteolysis.

$7 N D$ protein decreases the number of osteoclasts in the bone resorption lacunae. $\mathrm{H} \& \mathrm{E}$ and TRAP staining were used to visualize and count osteoclasts accumulating in the resorption lacunae. The results demonstrated that significantly more osteoclasts were detected in the LPS group compared with the PBS and 7ND groups, whereas the 7ND group showed no significant difference compared with the PBS group 
A
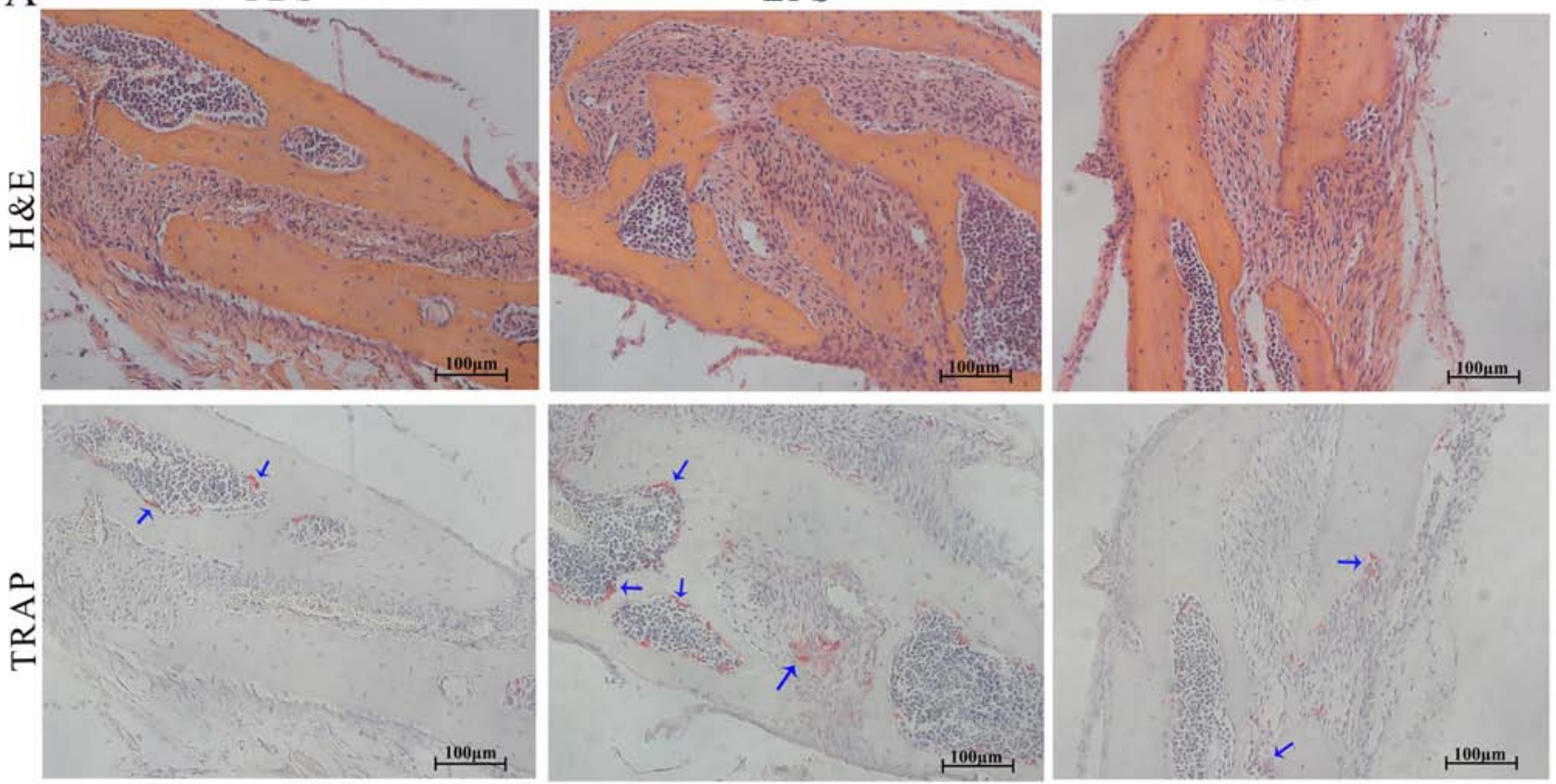

B
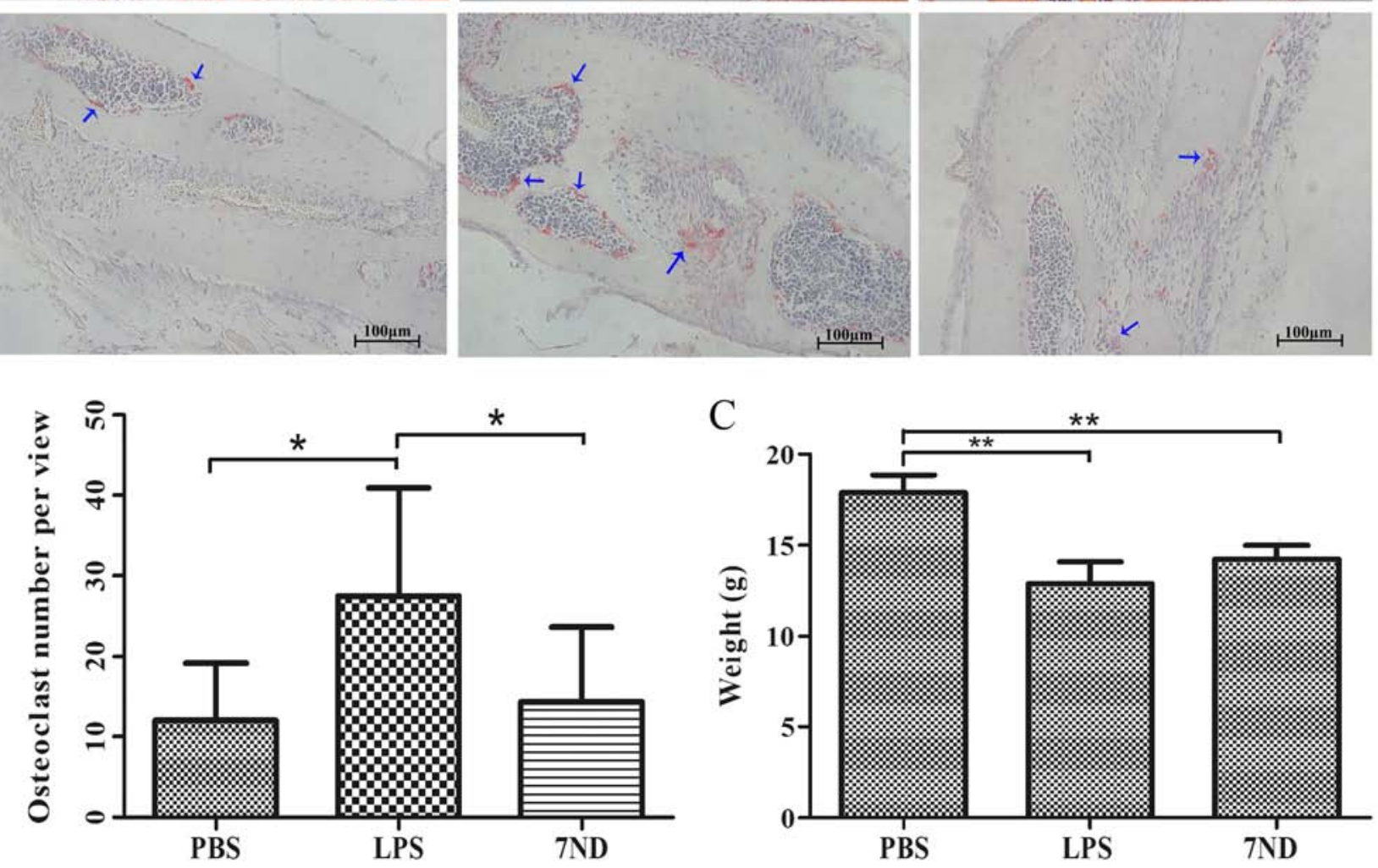

Figure 4. 7ND protein inhibits osteoclast accumulation in resorption lacunae. (A) Fewer osteoclasts were detected in the 7ND group, as assessed by H\&E and TRAP staining. Blue arrows indicate osteoclast-like cells. Scale bar, $100 \mu \mathrm{m}$. (B) Quantification of TRAP-positive cells was performed by analysing six randomly selected fields. (C) Mouse weight. $\mathrm{N}=6$ /group. ${ }^{*} \mathrm{P}<0.05 ;{ }^{* * *} \mathrm{P}<0.01$. H\&E, haematoxylin and eosin; TRAP, tartrate-resistant acid phosphatase; $7 \mathrm{ND}$, seven-amino acid truncated; LPS, lipopolysaccharide.

(Fig. 4A and B), in line with the $\mu$-CT results. As mice in the LPS and 7ND groups were injected with LPS, their weight was lower compared to the PBS group where mice were not injected with LPS (Fig. 4C).

\section{Discussion}

7ND protein is a mutant form of MCP-1 without the seven $\mathrm{N}$-terminal amino acids at positions 2-8. 7ND binds to the MCP-1 receptor on macrophages and functions as a dominant-negative inhibitor of MCP-1 $(6,10)$. MCP-1 plays a critical role in chemotaxis and inflammation, activating cell mobilization and upregulating a series of pro-inflammatory cytokines and chemokines (15). However, Yao et al (6) showed that 7ND protein treatment decreased not only MCP-1-induced migration of THP-1 cells in a dose-dependent manner, but also the THP-1 chemotactic effects of conditioned media from Raw 264.7 murine macrophages exposed to polymethylmethacrylate particles with and/or without LPS. In addition, the 7ND protein is able to bind to the MCP-1 receptor without inducing cell activation, and interference of the MCP-1-C-C motif chemokine receptor 2 ligand-receptor axis was able to decrease the release of inflammatory cytokines without adverse effects $(6,16)$.

The present results suggested that 7ND protein efficiently inhibited PBMCs differentiation into osteoclasts without influencing cell proliferation, in line with the results by Kim et al $(13,14)$. The present study has demonstrated the significant effects of 7ND protein in vitro, which is the first step for animal experiments in vivo. Previous studies identified that 7ND protein exhibited promising results in decreasing osteolysis. Morrison et al (17) found that 7ND blocked calmodulin 1 (CALM1), Jun proto-oncogene (JUN), AP-1 transcription factor subunit and nuclear factor of activated T cells 2 (NFATC2) induction in colony forming unit-granulocyte macrophages and inhibited human osteoclast differentiation at the molecular level. Yao et al (6) showed that 
7ND protein decreased macrophage migration and the release of inflammatory cytokines in a dose-dependent manner. Therefore, in the present study, the effects of 7ND protein on osteoclast differentiation were investigated, and F-actin ring formation and TRAP expression were examined.

Various previous studies demonstrated that 7ND protein treatment significantly decreased osteolysis induced by orthopaedic implant wear particles $(7,12,18)$. Wear particles are by-products of all joint replacements and are able to stimulate chronic inflammation, thus leading to osteolysis. Wear particles increase the production of pro-inflammatory chemokines, including MCP-1, TNF- $\alpha$, ILs and granulocyte-monocyte CSF (6). However, a study of the effect of 7ND protein on bacterial osteolysis has yet to be reported. It has been hypothesized that osteolysis induced by chronic Gram-negative bacterial infection underlies bone diseases, and it has been demonstrated that LPS is a major virulence factor found in the Gram-negative bacterial cell wall (19). LPS was identified to induce intracellular activation of mitogen-activated protein kinases 8 and 14, and nuclear factor $\kappa \mathrm{B}$ in macrophages and monocytes, thus promoting the release of pro-inflammatory cytokines that are recognized as key pathogens of inflammatory osteolytic diseases, such as osteomyelitis, septic arthritis, periodontitis and infection of orthopaedic implants $(20,21)$. Among various mouse models of osteolysis, an inflammation-induced bone loss model through LPS injection into the calvaria was used in the present study. Compared with other animal models (22-25), this model exhibits the advantage of accurate and reproducible injection into the calvaria between the ears of mice $(19,26,27)$. In addition, the present model is not affected by interferences caused by possible animal activity.

Inhibition of LPS-induced osteolysis is important to prevent bone destruction in infective bone diseases. Assessing the effects of novel agents on LPS-induced osteoclastogenesis should be studied in vivo using murine models. However, various studies established LPS-induced osteolysis models in multiple ways, and no standard protocols were described to determine the injection time in the murine model (22-27). To solve these problems, the present study established an LPS-induced bone loss mouse model. The present $\mu$-CT imaging results suggested that the percentage of the bone destruction area, indicating osteolysis, induced by high concentrations $(25 \mathrm{mg} / \mathrm{kg}$ ) of LPS lasted $>5$ days, whereas low concentrations $(5 \mathrm{mg} / \mathrm{kg})$ induced osteolysis for 1 day. The present results suggested that $25 \mathrm{mg} / \mathrm{kg}$ LPS is recommended for long-term experiments (5-7 days), whereas $5 \mathrm{mg} / \mathrm{kg} \mathrm{LPS}$ is recommended for short-term experiments (1-3 days). Based on the present findings, a long-term murine osteolysis model following treatment with $25 \mathrm{mg} / \mathrm{kg}$ LPS was used to determine the efficacy of local 7ND protein delivery in vivo.

Based on the inhibitory effects of 7ND protein on osteoclast differentiation in vitro, further in vivo experiments were performed. As assessed by $\mu$-CT imaging, injection of 7ND protein effectively mitigated LPS-induced bone loss. 7ND treatment significantly reduced bone osteolysis and maintained normal bone morphology. Quantitative analyses of $\mu$-CT images revealed that 7ND protein treatment increased the BMD, BV fraction and BV/TV compared with PBS. Histological analyses indicated that 7ND protein effectively reduced the number of osteoclasts. Importantly, $\mu$-CT and histological analyses showed no significant differences between local delivery of 7ND protein in the LPS-induced-osteolysis model and PBS in normal mice, demonstrating that 7ND protein significantly inhibited LPS-induced osteolysis. Subcutaneous injection of 7ND protein may have an antiresorptive effect on LPS-induced bone loss, and the molecular mechanism of 7ND protein requires further investigation in vivo. Nabeshima et al (18) showed that $7 \mathrm{ND}$ protein coating decreased the number of infiltrating macrophages in a continuous polyethylene particle infusion model. Therefore, further investigation is required to clarify the mechanism of 7ND protein on LPS-induced bone loss. Further in vivo study may identify the direct effects of 7ND protein on osteoclast differentiation and its indirect anti-inflammatory effects.

In the present study, 7ND protein efficiently inhibited osteoclast differentiation in PBMCs in vitro and significantly reduced LPS-induced osteolysis in vivo. Morrison et al (17) showed that colony forming unit-granulocyte macrophages increased by 1,000-fold the differentiation potential of MCP-1 within $24 \mathrm{~h}$ of TNFSF11 treatment, and 7ND inhibited osteoclast differentiation by suppressing the expression levels of CALM1, JUN and NFATC2. Therefore, in the present study, it was hypothesized that the 7ND protein inhibited human osteoclast formation at an early stage of differentiation by interfering with the expression of pro-inflammatory cytokines, thus inhibiting the signalling pathways involved in inflammation-mediated stimulation. However, the molecular mechanisms underlying 7ND protein function on the inhibition of osteoclast differentiation requires further investigation.

In conclusion, the present study suggested the potential of the 7ND protein in inhibiting osteoclast differentiation and reducing bone loss. The present results suggested that the 7ND protein may represent a novel therapeutic candidate to treat various osteoclast-associated bone diseases.

\section{Acknowledgements}

Not applicable.

\section{Funding}

The present work was supported by The Natural Science Foundation of Guangdong Province (grant no.2017A030313713) and The National Natural Science Foundation of China (grant nos. 81870750 and 81500839$)$.

\section{Availability of data and materials}

All data generated or analysed during the present study are included in this published article.

\section{Authors' contributions}

HJ and QG conceived the present study. WL, JQ, YL and JL performed the experiments. WL and JQ designed the study, performed the experiments, analysed the data and wrote the manuscript. WL and JQ contributed equally. All authors read and approved the final manuscript. 


\section{Ethics approval and consent to participate}

All experiments were approved by The Sun Yat-sen University Ethics Committee (ethics certificate nos. IACUC-DB-17-1105 and IACUC-DD-17-1112).

\section{Patient consent for publication}

Not applicable.

\section{Competing interests}

The authors declare that they have no competing interests.

\section{References}

1. Lenertz LY, Baughman CJ, Waldschmidt NV, Thaler R and van Wijnen AJ: Control of bone development by P2X and P2Y receptors expressed in mesenchymal and hematopoietic cells. Gene 570: 1-7, 2015

2. Nakashima T and Takayanagi H: New regulation mechanisms of osteoclast differentiation. Ann N Y Acad Sci 1240: E13-E18, 2011

3. Boyce BF: Advances in the regulation of osteoclasts and osteoclast functions. J Dent Res 92: 860-867, 2013.

4. Muto A, Mizoguchi T, Udagawa N, Ito S, Kawahara I, Abiko Y, Arai A, Harada S, Kobayashi Y, Nakamichi Y, et al: Lineage-committed osteoclast precursors circulate in blood and settle down into bone. J Bone Miner Res 26: 2978-2990, 2011.

5. Ko SY: Myricetin suppresses LPS-induced MMP expression in human gingival fibroblasts and inhibits osteoclastogenesis by downregulating NFATc1 in RANKL-induced RAW 264.7 cells. Arch Oral Biol 57: 1623-1632, 2012.

6. Yao Z, Keeney M, Lin TH, Pajarinen J, Barcay K, Waters H, Egashira K, Yang F and Goodman S: Mutant monocyte chemoattractant protein 1 protein attenuates migration of and inflammatory cytokine release by macrophages exposed to orthopedic implant wear particles. J Biomed Mater Res A 102: 3291-3297, 2014.

7. Jiang X, Sato T, Yao Z, Keeney M, Pajarinen J, Lin TH, Loi F, Egashira K, Goodman S and Yang F: Local delivery of mutant CCL2 protein-reduced orthopaedic implant wear particle-induced osteolysis and inflammation in vivo. J Orthop Res 34: 58-64, 2016

8. Chen W, Foo SS, Taylor A, Lulla A, Merits A, Hueston L, Forwood MR, Walsh NC, Sims NA, Herrero LJ and Mahalingam S: An inhibitor of monocyte chemotactic protein synthesis, protects against bone loss induced by chikungunya virus infection. J Virol 89: 581-593, 2015.

9. Sierra-Filardi E, Nieto C, Domínguez-Soto A, Barroso R, Sánchez-Mateos P, Puig-Kroger A, López-Bravo M, Joven J, Ardavín C, Rodríguez-Fernández JL, et al: CCL2 shapes macrophage polarization by GM-CSF and M-CSF: Identification of CCL2/CCR2-dependent gene expression profile. J Immunol 192: 3858-3867, 2015.

10. Zhang Y, Ernst CA and Rollins BJ: MCP-1: Structure/activity analysis. Methods 10: 93-103, 1996.

11. Keeney M, Waters H, Barcay K, Jiang X, Yao Z, Pajarinen J, Egashira K, Goodman SB and Yang F: Mutant MCP-1 protein delivery from layer-by-layer coatings on orthopedic implants to modulate inflammatory response. Biomaterials 34: 10287-10295, 2013.

12. Luo S, Zhou C, Zhang J, Chen M, Li H, Zheng S and Quan J: Mutant monocyte chemoattractant protein-1 protein (7ND) inhibits osteoclast differentiation and reduces oral squamous carcinoma cell bone invasion. Oncol Lett 15: 7760-7768, 2018

13. Kim MS, Day CJ, Selinger CI, Magno CL, Stephens SR and Morrison NA: MCP-1-induced human osteoclast-like cells are tartrate-resistant acid phosphatase, NFATc1, and calcitonin receptor-positive but require receptor activator of NFkappaB ligand for bone resorption. J Biol Chem 281: 1274-1285, 2006.
14. Kim MS, Day CJ and Morrison NA: MCP-1 is induced by receptor activator of nuclear factor- $\{$ kappa\}B ligand, promotes human osteoclast fusion, and rescues granulocyte macrophage colony-stimulating factor suppression of osteoclast formation. J Biol Chem 280: 16163-16169, 2005.

15. Gibon E, Ma T, Ren PG, Fritton K, Biswal S, Yao Z, Smith L and Goodman SB: Selective inhibition of the MCP-1-CCR2 ligand-receptor axis decreases systemic trafficking of macrophages in the presence of UHMWPE particles. J Orthop Res 30: 547-553, 2012 .

16. Zhang Y and Rollins BJ: A dominant negative inhibitor indicates that monocyte chemoattractant protein 1 functions as a dimer. Mol Cell Biol 15: 4851-4855, 1995.

17. Morrison NA, Day CJ and Nicholson GC: Dominant negative MCP-1 blocks human osteoclast differentiation. J Cell Biochem 115: 303-312, 2014.

18. Nabeshima A,Pajarinen J,Lin TH, Jiang X, Gibon E, Córdova LA, Loi F, Lu L, Jämsen E, Egashira K, et al: Mutant CCL2 protein coating mitigates wear particle-induced bone loss in a murine continuous polyethylene infusion model. Biomaterials 117: 1-9, 2017.

19. Zhou X, Zhang C, Wang X, An B, Zhang P and Zhu Z: Berberine inhibits lipopolysaccharide- and polyethylene particle-induced mouse calvarial osteolysis in vivo. J Surg Res 73: e47-e52, 2012.

20. Hou GQ, Guo C, Song GH, Fang N, Fan WJ, Chen XD, Yuan L and Wang ZQ: Lipopolysaccharide (LPS) promotes osteoclast differentiation and activation by enhancing the MAPK pathway and COX-2 expression in RAW264.7 cells. Int J Mol Med 32: 503-510, 2013.

21. Robertson Remen KM, Lerner UH, Gustafsson JA and Andersson G: Activation of the liver X receptor- $\beta$ potently inhibits osteoclastogenesis from lipopolysaccharide-exposed bone marrow-derived macrophages. J Leukoc Biol 93: 71-82, 2013.

22. Baek JM, Kim JY, Ahn SJ, Cheon YH, Yang M, Oh J and Choi MK: Dendrobium moniliforme exerts inhibitory effects on both receptor activator of nuclear factor kappa-B ligand-Mediated osteoclast differentiation in vitro and lipopolysaccharide-induced bone erosion in vivo. Molecules 21: 295, 2016.

23. Baek JM, Kim JY, Cheon YH, Park SH, Ahn SJ, Yoon KH, Oh J and Lee MS: Aconitum pseudo-laeve var. erectum inhibits receptor activator of nuclear factor kappa-B ligand-induced osteoclastogenesis via the c-Fos/nuclear factor of activated T-Cells, cytoplasmic 1 signaling pathway and prevents lipopolysaccharide-induced bone loss in mice. Molecules 19: 11628-11644, 2014.

24. Baek JM, Kim JY, Jung Y, Moon SH, Choi MK, Kim SH, Lee MS, Kim I and Oh J: Mollugin from rubea cordifolia suppresses receptor activator of nuclear factor- $\kappa \mathrm{B}$ ligand-induced osteoclastogenesis and bone resorbing activity in vitro and prevents lipopolysaccharide-induced bone loss in vivo. Phytomedicine 22: 27-35, 2015.

25. Kim JY, Baek JM, Ahn SJ, Cheon YH, Park SH, Yang M, Choi MK and Oh J: Ethanolic extract of schizonepeta tenuifolia attenuates osteoclast formation and activation in vitro and protects against lipopolysaccharide-induced bone loss in vivo. BMC Complement Altern Med 16: 301, 2016.

26. Li L, Khansari A, Shapira L, Graves DT and Amar S: Contribution of interleukin-11 and prostaglandin(s) in lipopolysaccharide-induced bone resorption in vivo. Infect Immun 70: 3915-3922, 2002.

27. Espirito Santo AI, Ersek A, Freidin A, Feldmann M, Stoop AA and Horwood NJ: Selective inhibition of TNFR 1 reduces osteoclast numbers and is differentiated from anti-TNF in a LPS-driven model of inflammatory bone loss. Biochem Biophys Res Commun 464: 1145-1150, 2015.

This work is licensed under a Creative Commons Attribution-NonCommercial-NoDerivatives 4.0 International (CC BY-NC-ND 4.0) License. 